This item was submitted to Loughborough's Research Repository by the author.

Items in Figshare are protected by copyright, with all rights reserved, unless otherwise indicated.

\title{
Surface hopping dynamics including intersystem crossing using the algebraic diagrammatic construction method
}

PLEASE CITE THE PUBLISHED VERSION

https://doi.org/10.1063/1.4999687

PUBLISHER

AIP

VERSION

VoR (Version of Record)

LICENCE

CC BY-NC-ND 4.0

\section{REPOSITORY RECORD}

Mai, Sebastian, Felix Plasser, Mathias Pabst, Frank Neese, Andreas Kohn, and Leticia Gonzalez. 2019. "Surface Hopping Dynamics Including Intersystem Crossing Using the Algebraic Diagrammatic Construction Method". figshare. https://hdl.handle.net/2134/32126. 


\title{
Surface hopping dynamics including intersystem crossing using the algebraic diagrammatic construction method
}

\author{
Sebastian Mai, ${ }^{1, a)}$ Felix Plasser, ${ }^{1, b)}$ Mathias Pabst, ${ }^{2}$ Frank Neese, ${ }^{3}$ Andreas Köhn, ${ }^{2,4}$ \\ and Leticia González ${ }^{1}$ \\ ${ }^{1}$ Institute of Theoretical Chemistry, Faculty of Chemistry, University of Vienna, Währinger Str. 17, \\ 1090 Vienna, Austria \\ ${ }^{2}$ Institute of Physical Chemistry, University of Mainz, Duesbergweg 10, D-55099 Mainz, Germany \\ ${ }^{3}$ Max Planck Institute for Chemical Energy Conversion, Stiftstr. 34-36, D-45470 Mülheim an der Ruhr, Germany \\ ${ }^{4}$ Institute of Theoretical Chemistry, University of Stuttgart, Pfaffenwaldring 55, D-70569 Stuttgart, Germany
}

(Received 10 August 2017; accepted 20 October 2017; published online 13 November 2017)

\begin{abstract}
We report an implementation for employing the algebraic diagrammatic construction to second order $[\mathrm{ADC}(2)]$ ab initio electronic structure level of theory in nonadiabatic dynamics simulations in the framework of the SHARC (surface hopping including arbitrary couplings) dynamics method. The implementation is intended to enable computationally efficient, reliable, and easy-to-use nonadiabatic dynamics simulations of intersystem crossing in organic molecules. The methodology is evaluated for the 2-thiouracil molecule. It is shown that $\operatorname{ADC}(2)$ yields reliable excited-state energies, wave functions, and spin-orbit coupling terms for this molecule. Dynamics simulations are compared to previously reported results using high-level multi-state complete active space perturbation theory, showing favorable agreement. Published by AIP Publishing. https://doi.org/10.1063/1.4999687
\end{abstract}

\section{INTRODUCTION}

Intersystem crossing (ISC) is a fundamental photophysical process which can occur after a molecule is excited by the absorption of light. Specifically, during ISC the excited-state population is nonradiatively transferred between electronic states of different multiplicity, i.e., a change of the total spin of the electronic wave function occurs. ISC is usually contrasted with internal conversion (IC), which is a population transfer between states of the same multiplicity.

ISC plays an essential role in photoinduced processes ${ }^{1,2}$ in different areas of research. For example, in light harvesting, ${ }^{3,4}$ Organic light-emitting diode (OLED) design, ${ }^{5}$ magnetic data storage, ${ }^{6}$ and molecular electronics, ${ }^{7,8}$ ISC is often responsible for improving the efficiency of the application or even for enabling it in the first place. ISC is also relevant for several biological processes, like oxygen binding to proteins, ${ }^{9}$ DNA photodamage, ${ }^{10}$ or photodynamic therapy. ${ }^{11}$ While traditionally, ISC was regarded as much slower than IC-due to the fact that ISC is completely forbidden in a non-relativistic framework-nowadays it has been shown that ISC also occurs on ultrafast time scales in the pico- to femtosecond range. This is true not only for diverse transition metal complexes ${ }^{12-17}$ but also for small (bio-)organic molecules without very heavy atoms, like ketones ${ }^{18,19}$ or modified nucleobases. ${ }^{20-22}$

The theoretical description of ISC-especially ultrafast ISC - is very challenging. On the one hand, ISC is a relativistic effect and is mediated by spin-orbit couplings (SOCs). There are multiple challenges involved when doing

a)Electronic mail: sebastian.mai@univie.ac.at

b)Electronic mail: felix.plasser@univie.ac.at accurate relativistic quantum chemistry, for example, due to the (bi-)spinor nature of the wave functions, due to the negative-energy continuum of the eigenspectrum of the Dirac/Breit equations, or due to the high 1- and N-particle basis set requirements. ${ }^{23,24}$ On the other hand, it is often necessary to include the coupling between electronic and nuclear degrees of freedom. Whereas for slow ISC processes, it is possible to use perturbative approaches, e.g., Fermi's golden rule ${ }^{25}$ explicit dynamics simulations are required to simulate ultrafast ISC. It is possible to simulate ISC dynamics by means of grid-based quantum dynamics or multi-configurational time-dependent Hartree. ${ }^{26-28}$ However, these methods rely on a careful parameterization of the model used and are often severely hampered by the fact that they can only include a small number of nuclear degrees of freedom. Therefore, in the last few years, several groups have established nonadiabatic dynamics methods compatible with the on-the-fly computation of the potential energy surfaces (PESs). The surface hopping method ${ }^{29,30}$ has been shown to be particularly viable for this purpose, ${ }^{31-34}$ and also the related $a b$ initio multiple spawning ${ }^{35}$ has been applied. Over the last few years, some of us have devoted considerable effort to the SHARC (surface hopping including arbitrary couplings $)^{33,36}$ code, which is a freely available nonadiabatic dynamics package ${ }^{37}$ that allows performing IC and ISC dynamics with various electronic structure methods implemented in different quantum chemistry packages.

Nonadiabatic dynamics simulations for ISC rely on a proper choice of an electronic structure method because this choice affects the accuracy of the calculation, possibly more than any other simulation parameter. The chosen electronic structure method does not only need to provide accurate energies but it also has to produce energy gradients, nonadiabatic coupling terms, and SOCs to be compatible with surface 
hopping for ISC. At the same time, it has to be efficient enough to allow for the $10^{4}$ to $10^{5}$ single-point calculations required for a typical ensemble of trajectories. In the past, a number of different electronic structure methods were employed for such ISC-focused simulations. Semi-empirical methods offer a computationally efficient possibility ${ }^{31,38,39}$ although a careful parameterization of the Hamiltonian is required. Within $a b$ initio methods, one popular choice is the complete active space self-consistent field (CASSCF) method. ${ }^{40-43}$ Unfortunately, CASSCF lacks dynamical correlation. The application of multi-reference methods with dynamical correlation, like multi-state complete active space perturbation theory (MS-CASPT2) ${ }^{44-46}$ or multi-reference configuration interaction (MRCI), ${ }^{47-50}$ significantly improves the accuracy of the simulations but also dramatically increases their computational expense. Among the single-reference electronic structure methods, so far only time-dependent density functional theory (TDDFT) has been applied for ISC-focused dynamics simulations. ${ }^{34,51}$ Although here computational efficiency and dynamical correlation are in principle given, in TDDFT the choice of the appropriate exchange-correlation functional can strongly affect the quality of the results.

In order to expand the range of applicable electronic structure methods for ISC dynamics simulations, we have extended the nonadiabatic dynamics package SHARc ${ }^{33,36,37}$ to use the ab initio algebraic diagrammatic construction at second order [ADC(2)] method from the TuRBomole electronic structure suite. ${ }^{52}$ Previously, nonadiabatic dynamics simulations with this method were only possible within one multiplicity, i.e., only for IC but not ISC. ${ }^{53}$ The present implementation allows for the computation of nonadiabatic coupling terms within the singlet and triplet manifolds, as well as SOCs between excited singlet and triplet states. It is thus possible to model IC within the singlet and triplet manifolds and ISC between them. Currently, the computation of triplet-triplet SOCs is not supported. Triplet-triplet SOCs (e.g., routinely available in multi-configurational methods) can be of similar magnitude as singlet-triplet SOCs; however, their importance for nonadiabatic dynamics is often minor as triplet-triplet IC is dominated by nonadiabatic couplings. Only if the nonadiabatic couplings are very weak (e.g., symmetry forbidden ${ }^{47}$ ) or the SOCs are very large (e.g., transition metal complexes ${ }^{27}$ ), triplet-triplet SOCs will notably influence the dynamics. In organic molecules, it can be expected that triplet-triplet SOCs are not of prime relevance.

The idea of computing SOCs with ADC(2) (and CC2) has been initially introduced by Pabst and Köhn ${ }^{54}$ in 2011. This methodology, as implemented in Turbomole, is used here. More recently, Krauter et al. ${ }^{55}$ and Helmich-Paris et al. ${ }^{56}$ reported on further implementations of SOCs for $\mathrm{ADC}(2)$ and CC2, respectively. The implementation by Krauter et al. ${ }^{55}$ in Q-CHEM employs an efficient algorithm for computing exact state-to-state transition moments ${ }^{57}$ using the intermediatestate representation. ${ }^{58}$ In contrast, $\operatorname{ADC}(2)$ state-to-state transition moments in the TURBomole implementation are computed approximately by restricting the terms to those appearing in the closely related $\mathrm{CC} 2$ response theory. ${ }^{59}$ Furthermore, TURbomole features a powerful resolution-of-the-identity (RI) approximation, ${ }^{60}$ which allows for extremely efficient

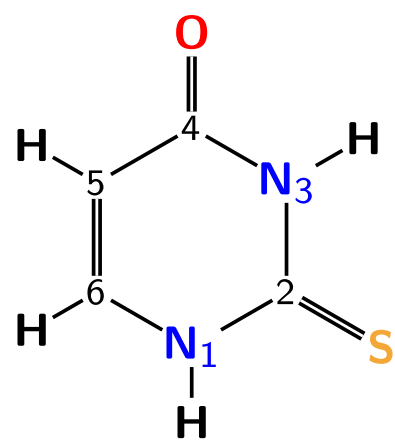

FIG. 1. Structure of the most stable tautomer of 2-thiouracil (2TU) and atom numbering.

excited-state computations. We note here that in addition to specific SOC implementations, there also exist general SOC implementations, like the recent PySOC, ${ }^{61}$ which could in principle be employed in nonadiabatic dynamics simulations like the ones targeted here.

The power of the newly implemented method is demonstrated for the case of 2-thiouracil (2TU, Fig. 1), an analog of uracil where one oxygen atom has been replaced by sulfur. The excited-state dynamics of 2TU was intensively studiedboth experimentally ${ }^{62-64}$ and theoretically ${ }^{45,65-67}$-in the last few years due to its biological relevance and the fact that it exhibits ultrafast $(<1 \mathrm{ps})$ ISC with nearly $100 \%$ quantum yield. Here, we compare excited-state dynamics obtained with the new RI-ADC(2) implementation to previously reported MS-CASPT2-based dynamics simulations. ${ }^{45}$ The two methods agree in terms of the overall ISC times although they differ in some of the mechanistic details. At the same time, RI-ADC(2) dynamics allows for dramatic computational savings.

\section{METHODS}

\section{A. Ab initio surface hopping dynamics}

The excited-state dynamics simulations with SHARc are performed in a fully diagonalized state basis, as detailed in Ref. 33 and briefly summarized below. In the case of ISC dynamics, the total electronic Hamiltonian needs to contain the SOCs. Due to the complexity of full 2- or 4-component electronic structure methods, SHARc is based on a perturbational ansatz, where the Hamiltonian is split into the molecular Coulomb Hamiltonian (MCH) and the spin-orbit Hamiltonian,

$$
\hat{H}_{\text {total }}=\hat{H}_{\mathrm{MCH}}+\hat{H}_{\mathrm{SOC}} .
$$

The electronic structure problem is first solved for $\hat{H}_{\mathrm{MCH}}$, yielding a small set of lowest-energy states (called the $\mathrm{MCH}$ states here) of different multiplicities, e.g., singlets and triplets. In the space of these states, the matrix representation of $\hat{H}_{\text {total }}$ in the basis of the $\mathrm{MCH}$ states (written as $\mathbf{H}^{\mathrm{MCH}}$ ) is computed and diagonalized to yield the diagonal energies and eigenvectors,

$$
\mathbf{H}^{\text {diag }}=\mathbf{U}^{\dagger} \mathbf{H}^{\mathrm{MCH}} \mathbf{U}
$$

where $\mathbf{U}$ is the transformation matrix between the $\mathrm{MCH}$ and diagonal bases. 
The diagonal entries of $\mathbf{H}^{\text {diag }}$ are the eigenenergies of the total Hamiltonian, which are the energies on which the nuclear dynamics is simulated. The nuclear motion is governed by the classical equation of motion, which is integrated by the velocity-Verlet algorithm. ${ }^{68}$ The required forces are calculated by transforming the gradients of the $\mathrm{MCH}$ states into the diagonal basis.

As with all surface hopping methods, ${ }^{29,30}$ the forces are evaluated for the current active state. In order to find the active state, the total electronic wave function is written as a linear combination of the diagonal states,

$$
\left|\Psi^{\mathrm{el}}(t)\right\rangle=\sum_{\alpha} c_{\alpha}(t)\left|\Psi_{\alpha}^{\mathrm{diag}}(t)\right\rangle,
$$

which is propagated from time $t$ to time $t+\Delta t$ by

$$
\mathbf{c}^{\mathrm{diag}}(t+\Delta t)=\mathbf{U}^{\dagger}(t+\Delta t) \mathbf{P}^{\mathrm{MCH}}(t+\Delta t, t) \mathbf{U}(t) \mathbf{c}^{\mathrm{diag}}(t) .
$$

The propagator matrix $\mathbf{P}^{\mathrm{MCH}}(t+\Delta t, t)$ can be computed by appropriately integrating over the Hamiltonian matrix and the nonadiabatic coupling terms from $t$ to $t+\Delta t$. Instead of using the nonadiabatic couplings, the propagator matrix could also be calculated from the overlap matrix $\left(S_{I J}=\left\langle\Psi_{I}(t) \mid \Psi_{J}(t+\Delta t)\right\rangle\right)$ using the local diabatization method. ${ }^{69,70}$ From the change of the wave function coefficients $\mathbf{c}^{\text {diag }}$, the active state of the next time step is found stochastically. ${ }^{33}$

From the abovesaid, it follows that several properties need to be calculated at each simulation time step: (i) energies, (ii) gradients, (iii) SOCs, and (iv) nonadiabatic couplings or overlap matrices. For setting up initial conditions (or explicit interactions with a laser field), dipole and transition dipole moments are also needed. Any quantum chemistry method/program used together with SHARc needs to provide these quantities in an efficient and consistent manner.

\section{B. ADC(2) excitation energies and transition densities}

In $\mathrm{ADC}(2)$ theory, ${ }^{71,72}$ excitation energies are obtained from an eigenvalue equation of the form

$$
\mathbf{A R}^{I}=\mathbf{R}^{I} \omega_{I},
$$

where the eigenvectors consist of a single excitation part $\mathbf{R}_{1}$ (associated with single excitation operators $\hat{\tau}_{\rho_{1}}$ relative to the Hartree-Fock reference state $\left.\left|\Phi_{0}\right\rangle\right)$ and a double excitation part $\mathbf{R}_{2}$ (double excitation operators $\hat{\tau}_{\rho_{2}}$ ). Here, we use the general indices $\rho_{1}, \sigma_{1}$, and $\rho_{2}, \sigma_{2}$ to refer to single and double excitation manifolds, respectively. An explicit representation for singlet and triplet excitations is given in the Appendix. The symmetric matrix $\mathbf{A}$ can be written in blocked form as

$$
\mathbf{A}=\left(\begin{array}{ll}
A_{\rho_{1} \sigma_{1}} & A_{\rho_{1} \sigma_{2}} \\
A_{\rho_{2} \sigma_{1}} & A_{\rho_{2} \sigma_{2}}
\end{array}\right)
$$

with

$$
\begin{aligned}
A_{\rho_{1} \sigma_{1}} & =\hat{\mathcal{S}}_{\rho_{1} \sigma_{1}}\left\langle\Phi_{0}\left|\hat{\tau}_{\rho_{1}}^{\dagger}\left[\hat{H}+\left[\hat{H}, \hat{T}_{2}\right], \hat{\tau}_{\sigma_{1}}\right]\right| \Phi_{0}\right\rangle, \\
A_{\rho_{1} \sigma_{2}} & =\left\langle\Phi_{0}\left|\hat{\tau}_{\rho_{1}}^{\dagger}\left[\hat{H}, \hat{\tau}_{\sigma_{2}}\right]\right| \Phi_{0}\right\rangle \\
A_{\rho_{2} \sigma_{1}} & =\left\langle\Phi_{0}\left|\hat{\tau}_{\rho_{2}}^{\dagger}\left[\hat{H}, \hat{\tau}_{\sigma_{1}}\right]\right| \Phi_{0}\right\rangle \\
A_{\rho_{2} \sigma_{2}} & =\left\langle\Phi_{0}\left|\hat{\tau}_{\rho_{2}}^{\dagger}\left[\hat{F}, \hat{\tau}_{\sigma_{2}}\right]\right| \Phi_{0}\right\rangle
\end{aligned}
$$

Here, $\hat{H}$ is the molecular electronic Hamiltonian and $\hat{F}$ is the Fock operator corresponding to the reference determinant $\left|\Phi_{0}\right\rangle . \hat{T}_{2}$ is the pair correlation cluster operator, determined from Møller-Plesset second-order perturbation (MP2) theory, and $\hat{\mathcal{S}}_{\rho_{1} \sigma_{1}}$ is a symmetrization operator: $\hat{\mathcal{S}}_{\rho_{1} \sigma_{1}} A_{\rho_{1} \sigma_{1}}=$ $\frac{1}{2}\left(A_{\rho_{1} \sigma_{1}}+A_{\sigma_{1} \rho_{1}}\right)$. In order to arrive at potential energy surfaces of excited states, the total energy of a state $I$ is defined as $E_{I}=E_{0}^{\mathrm{MP} 2}+\omega_{I}$. In the course of this work, we view $\operatorname{ADC}(2)$ as a simplified variant of the $\mathrm{CC} 2$ method. $^{73}$ The transition between the methods is easily accomplished by replacing the CC2 ground state equations by MP2 (effectively setting the $\hat{T}_{1}$ cluster operator to zero) and symmetrization of the matrix A. For details of the implementation, we refer to the original studies on $\mathrm{CC} 2$ using the resolution-of-the-identity (RI) approximation for fast evaluation of the two-electron repulsion integrals. ${ }^{60}$ The particular feature of this implementation is a recasting of the eigenvalue problem [Eq. (5)] into an effective (energy-dependent) eigenvalue problem for single excitations $\mathbf{R}_{1}$ only. The double excitation contribution can always be computed from the known $\mathbf{R}_{1}$ amplitudes; thus, $O\left(N^{4}\right)$ scaling storage of doubles' amplitudes is fully avoided. ${ }^{60}$

In the course of the present work, we need to define transition matrix elements between electronic ground or excited singlet states and triplet states. To this end, we again start from the usual response theory for coupled-cluster theory within the $\mathrm{CC} 2$ context and derive the ADC(2) expressions using the simplifications as explained in the previous paragraph. We note that this approach deviates from the true ADC(2) philosophy, which would require parts of the second-order ground state wave function in order to arrive at matrix elements that are fully correct through second-order perturbation theory. ${ }^{55}$ For the definition and the general outline of computing transition densities between the electronic ground state and excited states and between excited states within the $\mathrm{CC} 2$ context, we refer to Refs. 74 and 59. In the spin-orbital formulation, these expressions are easily generalized to triplet transition densities, i.e., transition densities of the spin-excitation operator $\hat{\mathbf{E}}_{p q}^{\text {spin }}$ whose Cartesian components are given as ${ }^{75}$

$$
\begin{aligned}
& \hat{E}_{p q}^{x}=\frac{1}{2}\left(\hat{a}_{p}^{\dagger} \hat{a}_{\bar{q}}+\hat{a}_{\bar{p}}^{\dagger} \hat{a}_{q}\right), \\
& \hat{E}_{p q}^{y}=\frac{\mathrm{i}}{2}\left(\hat{a}_{\bar{p}}^{\dagger} \hat{a}_{q}-\hat{a}_{p}^{\dagger} \hat{a}_{\bar{q}}\right), \\
& \hat{E}_{p q}^{z}=\frac{1}{2}\left(\hat{a}_{p}^{\dagger} \hat{a}_{q}-\hat{a}_{\bar{p}}^{\dagger} \hat{a}_{\bar{q}}\right) .
\end{aligned}
$$

Here, $\hat{a}_{p}^{\dagger}$ and $\hat{a}_{p}$ are the usual creation and annihilation operators and the bar is used to indicate the orbitals of $\beta$ spin. Here and in the following, $p$ and $q$ are the indices of general (occupied and virtual) spatial orbitals. As the implementation treats only the $M_{S}=0$ component of the triplet state explicitly, only transition moments of the $\hat{E}_{p q}^{z}$ component can be directly computed. The other components, however, are easily obtained by the Wigner-Eckart theorem (for details, see, e.g., Ref. 55).

A special feature of the present implementation is the use of a spin-adapted basis for the triplet operators, allowing the use of the fast spin-adapted code for the ground state 
of closed-shell systems. The corresponding expressions for triplet transition densities are given in the Appendix.

\section{Spin-orbit couplings}

The SOC terms are defined here as the matrix elements of the Breit-Pauli spin-orbit Hamiltonian ${ }^{24}$

$$
\begin{aligned}
\widehat{H}_{\mathrm{SO}, \mathrm{BP}}= & \frac{1}{2 c^{2}} \sum_{i=1}^{n_{\mathrm{el}}} \sum_{K=1}^{n_{\mathrm{nuc}}} \frac{Z_{K}\left(\mathbf{r}_{i K} \times \mathbf{p}_{i}\right) \cdot \mathbf{s}_{i}}{r_{i K}^{3}} \\
& -\frac{1}{2 c^{2}} \sum_{i, j \neq i}^{n_{\mathrm{el}}} \frac{\left(\mathbf{r}_{i j} \times \mathbf{p}_{i}\right) \cdot \mathbf{s}_{i}}{r_{i j}^{3}} \\
& +\frac{1}{c^{2}} \sum_{i, j \neq i}^{n_{\mathrm{el}}} \frac{\left(\mathbf{r}_{i j} \times \mathbf{p}_{i}\right) \cdot \mathbf{s}_{j}}{r_{i j}^{3}},
\end{aligned}
$$

which is given with respect to the Cartesian position $\mathbf{r}_{i K}, \mathbf{r}_{i j}$, momentum $\mathbf{p}_{i}$, and spin $\mathbf{s}_{i}$ operators of the different electrons. The terms in Eq. (14) are a one-electron term and the two-electron spin-same-orbit and spin-other-orbit interaction contributions. From a practical viewpoint, it is worth noting that the effort for computing a matrix element of $\widehat{H}_{\mathrm{SO}, \mathrm{BP}}$ exceeds that of the spin-free Hamiltonian due to the fact that more two-electron integrals have to be evaluated. However, the computational effort can be reduced by applying a mean field approach. ${ }^{75,76}$ For this purpose, an effective spin-orbit mean field (SOMF) operator is constructed, which is of the form

$$
\widehat{H}_{\mathrm{SOMF}}=\sum_{p q} \mathbf{f}_{p q} \cdot \hat{\mathbf{E}}_{p q}^{\mathrm{spin}}=\sum_{\zeta \in\{x, y, z\}} \sum_{p q} f_{p q}^{\zeta} \hat{E}_{p q}^{\zeta}
$$

with the spin-excitation operator $\hat{\mathbf{E}}_{p q}^{\mathrm{spin}}$ as defined in Eqs. (11)-(13).

The matrix elements $f_{p q}^{\zeta}$ are obtained by averaging the two-electron contributions over the Hartree-Fock one-particle density, in analogy to the definition of the Fock matrix, ${ }^{75}$

$$
f_{p q}^{\zeta}=h_{p q}^{\mathrm{SOC}, \zeta}+\sum_{k}\left(2 g_{k k p q}^{\mathrm{SOC}, \zeta}-3 g_{p k k q}^{\mathrm{SOC}, \zeta}+3 g_{q k k p}^{\mathrm{SOC}, \zeta}\right) .
$$

Here, $h_{p q}^{\mathrm{SOC}, \zeta}$ and $g_{p q r s}^{\mathrm{SOC}, \zeta}$ are the required one- and two-electron SOC integrals. The SOC between two wave functions $\Psi_{I}$ and $\Psi_{J}$ is in turn obtained through contraction with the spin-transition density matrix, ${ }^{75}$

$$
\begin{aligned}
\left\langle\Psi_{I}\left|\widehat{H}_{\mathrm{SOMF}}\right| \Psi_{J}\right\rangle & =\sum_{\zeta} \sum_{p q} D_{p q}^{I J, \zeta} f_{p q}^{\zeta}, \\
D_{p q}^{I J, \zeta} & =\left\langle\Psi_{I}\left|\hat{E}_{p q}^{\zeta}\right| \Psi_{J}\right\rangle .
\end{aligned}
$$

\section{Nonadiabatic interactions}

Nonadiabatic interactions are computed during the dynamics by a formalism initially proposed by HammesSchiffer and Tully ${ }^{77}$ and later refined by Persico and coworkers. ${ }^{69}$ In this approach, the inner product of the nonadiabatic coupling vector and the velocity, which determines the nonadiabatic transition probabilities, is not computed explicitly. Instead, the wave function overlap

$$
S_{I J}(t)=\left\langle\Psi_{I}(t) \mid \Psi_{J}(t+\Delta t)\right\rangle
$$

between the electronic wave functions computed at two different time steps is used in the computation of the propagator matrix in Eq. (4).

For computational efficiency and ease of implementation, the $\mathrm{ADC}(2)$ wave functions are approximated to be of the form of configuration interaction singles (CIS) wave functions using

$$
\left|\Psi_{I}\right\rangle=\sum_{i a} \frac{R_{i a}^{I}}{\left\|\mathbf{R}_{1}^{I}\right\|}\left|\Phi_{i a}\right\rangle,
$$

where $R_{i a}^{I}$ is the amplitude of the single excitation going from occupied orbital $i$ to virtual orbital $a$ and $\left|\Phi_{i a}\right\rangle$ is the corresponding Slater determinant. The idea of using approximate CIS wave functions for nonadiabatic dynamics has been previously used very successfully in the case of TDDFT, ${ }^{78-80}$ and the formalism has been extended to the case of $\mathrm{ADC}(2)$ more recently. ${ }^{53}$ The evaluation of Eq. (19) can be very costly even for the approximate wave functions. To allow for an efficient computation of this term, we adopted the optimized algorithm described in Ref. 81.

\section{COMPUTATIONAL DETAILS}

The computations are divided into two main parts. First, we present benchmark calculations, where we compute a few excited states at the Franck-Condon geometry of 2TU with $\operatorname{RI}-\operatorname{ADC}(2),{ }^{72,73} \mathrm{ADC}(3),{ }^{82,83}$ and MS-CASPT $2^{84}$ and use the two latter methods to scrutinize the accuracy of RI-ADC(2) for this molecule. These calculations were performed at the RI-MP2/def2-SVP optimized ground state minimum (see the supplementary material for coordinates). In the second part, we perform nonadiabatic dynamics simulations with SHARC coupled to RI-ADC(2).

The RI-ADC $(2)^{72,73}$ computations were performed using Turbomole $7.0^{52}$ and employed the def2-SVP basis set. ${ }^{85}$ For comparison, also a computation using the larger aug-cc-pVTZ basis set $^{86}$ was performed. The spin-orbit matrix elements were computed using the spin-orbit mean field (SOMF) formalism as implemented in the REL module of the ORCA 3.0.3 program package. ${ }^{76,87}$ The code was also tested for ORCA version 4.0.1. We use the seminumerical implementation of the Coulomb-like contributions to the spin-orbit mean-field integrals, while the exchange-like contributions are approximated by a one-center approximation. ${ }^{76}$ We note that future releases of TURBomole will also directly provide spin-orbit meanfield integrals, computed without further approximations, see Ref. 56.

ADC(3) computations, ${ }^{82,83}$ also employing the def2-SVP basis, were carried out with the Q-CHEM program package. ${ }^{88}$ In this case, a canonical implementation without RI approximation was used. Density matrices at the ADC(3) level were obtained by contracting the third order vectors with the second order intermediate-state representation. ${ }^{58}$

MS-CASPT2 ${ }^{84}$ computations were performed with Molcas $8.0^{89}$ using an active space of 14 electrons in 10 orbitals $\left(n_{S}, n_{O}, 5 \times \pi, 3 \times \pi^{*}\right.$; see the supplementary material for orbital plots) considering 6 singlet and 4 triplet states, i.e., MS(6,4)-CASPT2 $(14,10)$. The orbitals for the present 
computation were obtained by a state-averaged complete active space self-consistent field (SA-CASSCF) ${ }^{90}$ computation using the same active space and state-averaging. For these computations, the SVP basis set of Molcas was used, which is identical to def2-SVP in the case of 2TU. Following Ref. 91, for this rather small basis set, we use an IPEA shift of zero, ${ }^{92}$ which produces energies in good agreement with MS-CASPT2 calculations using much larger basis sets (up to quadruple$\zeta) .{ }^{67}$ Spin-orbit matrix elements were computed using the state interaction method combined with an atomic meanfield Hamiltonian. ${ }^{93,94}$ Note that our previous calculations for $2 \mathrm{TU}^{45}$ were performed with $\mathrm{MS}(3,3)$-CASPT2 $(12,9)$.

The produced wave functions were analyzed by visualizing natural transition orbitals. ${ }^{95}$ To this end, the TheoDORE 1.5 program package ${ }^{96-98}$ was used for the analysis of the Turbomole computations, whereas integrated analysis modules were employed for the results of the $\mathrm{Q}-\mathrm{CHEM}^{97}$ and MoLCAs $^{99}$ program packages.

Dynamics simulations were performed at the same RI-ADC(2)/def2-SVP level of theory as the benchmark excited-state calculations. Nonadiabatic interaction terms were computed by means of wave function overlaps, ${ }^{81}$ which were used in the local diabatization procedure to propagate the wave function. ${ }^{69,70}$ In preparation of the dynamics simulations, 200 initial geometries were sampled from a Wigner distribution of the ground state harmonic oscillator, based on a frequency calculation at the MP2/cc-pVDZ level of theory (as in Ref. 45). For each geometry, a single point calculation at the RI-ADC(2)/def2-SVP level of theory was performed, and the resulting excitation energies and oscillator strengths were used to simulate the absorption spectrum of 2TU (see below). The energies and oscillator strengths were employed to stochastically select bright initial states ${ }^{100}$ in the 3.9-4.3 eV energy window, which yielded 32 initial conditions for trajectories (18 starting in $S_{2}$ and 14 in $S_{3}$ ). The trajectories were propagated with SHARc, ${ }^{33,36,37}$ considering 4 singlet and 3 triplet states, for $1000 \mathrm{fs}$ with a 0.5 fs nuclear time step and a 0.02 fs electronic time step. An energy-based decoherence scheme was applied to the diagonal states. ${ }^{101}$ For the analysis, out of 32 trajectories, 3 were neglected because they showed a ring opening of 2TU, and RI-ADC(2) is not expected to provide reliable results in this situation due to the small $S_{1}-S_{0}$ energy gap. The results of the analysis of the 29 remaining trajectories are presented below.

\section{RESULTS AND DISCUSSION}

\section{A. Vertical excitations and SOC terms}

The purpose of this section is to evaluate the overall ability of the RI-ADC(2) method to compute excited state energies and wave functions at the Franck-Condon region and to specifically evaluate the SOC terms. In Table I, the main results of the RI-ADC(2)/def2-SVP computations at the ground-state minimum are presented and compared to two higher-level reference methods: ADC(3)/def2-SVP and MS(6,4)-CASPT2(14,10)/def2-SVP. For RI-ADC(2) also the results using the larger aug-cc-pVTZ basis set are given (in parentheses). For the double- $\zeta$ calculations, the corresponding natural transition orbitals ${ }^{95,97}$ are given in the supplementary material.

With all methods, the $S_{1}$ state is an excitation from the $n$ orbital on the S atom (denoted as $n_{S}$, see Ref. 67 for a more detailed discussion of the involved orbitals) to the $\pi^{*}$ orbital located between the S atom and C2 atom $\left(\pi_{2}^{*}\right)$. This state is located slightly below $4 \mathrm{eV}$ for RI-ADC(2) and MS-CASPT2 and slightly above this value for ADC(3). The next singlet excitation at the RI-ADC(2) level, denoted as ${ }^{1} n_{O} \pi_{6}^{*}$, originates from the $n$ orbital on the $\mathrm{O}$ atom and goes into a $\pi^{*}$ orbital located on the $\mathrm{C} 4, \mathrm{C} 5$, and $\mathrm{C} 6$ atoms (cf. Fig. 1). Its energy is around $4.7 \mathrm{eV}$ at the RI-ADC(2) and MS-CASPT2 levels, whereas it is significantly higher $(5.19 \mathrm{eV})$ at the ADC(3) level. For all the three methods, this state is distinguished by a particularly small dipole moment, around $2 \mathrm{D}$. Two bright $\pi \pi^{*}$ states follow, where in both cases, the donor orbital is a $\pi$ orbital predominantly located on the $\mathrm{S}$ atom $\left(\pi_{S}\right)$, while the acceptor is a $\pi^{*}$ orbital delocalized over the whole system, see Figs. S4 and S5 of the supplementary material (hence, we simply write " $\pi^{*}$ " in the table, without orbital index). The two resulting ${ }^{1} \pi_{S} \pi^{*}$ states are located at $4.8 \mathrm{eV}$ and

TABLE I. Vertical excitation energies $(\Delta E, \mathrm{eV})$, oscillator strengths $(f)$, and dipole moments $(\mu, \mathrm{D})$ at the MP2optimized ground-state minimum of $2 \mathrm{TU}$ computed at the RI-ADC(2)/def2-SVP, ADC(3)/def2-SVP, and MS(6,4)CASPT2(14,10)/def2-SVP levels of theory.

\begin{tabular}{|c|c|c|c|c|c|c|c|c|c|}
\hline \multirow[b]{2}{*}{ State $^{a}$} & \multicolumn{3}{|c|}{ RI-ADC(2) } & \multicolumn{3}{|c|}{$\mathrm{ADC}(3)$} & \multicolumn{3}{|c|}{ MS-CASPT2 } \\
\hline & $\Delta E^{\mathrm{b}}$ & $f$ & $\mu$ & $\Delta E$ & $f$ & $\mu$ & $\Delta E$ & $f$ & $\mu$ \\
\hline${ }^{1} n_{S} \pi_{2}^{*}$ & $3.90(3.75)$ & 0.000 & 4.5 & 4.15 & 0.000 & 4.9 & 3.81 & 0.000 & 5.1 \\
\hline${ }^{1} n_{O} \pi_{6}^{*}$ & $4.74(4.58)$ & 0.000 & 1.5 & 5.19 & 0.000 & 1.7 & 4.64 & 0.000 & 2.2 \\
\hline${ }^{1} \pi_{S} \pi^{*}$ & $4.78(4.42)$ & 0.377 & 4.5 & 4.86 & 0.253 & 5.6 & 4.32 & 0.421 & 5.4 \\
\hline $2^{1} \pi_{S} \pi^{*}$ & $5.28(4.92)$ & 0.107 & 4.6 & 5.33 & 0.227 & 4.7 & 4.99 & 0.353 & 4.7 \\
\hline${ }^{1} n_{S} \pi_{6}^{*}$ & $5.58(5.28)$ & 0.000 & 7.6 & 5.98 & 0.000 & 9.0 & 5.19 & 0.001 & 4.0 \\
\hline${ }^{3} \pi_{S} \pi_{2}^{*}$ & $3.50(3.42)$ & & 3.99 & 3.37 & & 3.77 & 3.34 & & 3.80 \\
\hline${ }^{3} n^{S} \pi_{2}^{*}$ & $3.75(3.64)$ & & 4.70 & 4.00 & & 4.93 & 3.85 & & 4.56 \\
\hline${ }^{3} \pi \pi^{*}$ & $4.07(3.98)$ & & 3.42 & 3.86 & & 3.65 & 3.85 & & 3.31 \\
\hline${ }^{3} n_{O} \pi_{6}^{*}$ & $4.51(4.40)$ & & 1.92 & 4.95 & & 2.16 & 4.69 & & 3.03 \\
\hline
\end{tabular}

${ }^{a}$ The orbital nomenclature is given in Fig. S1 of the supplementary material.

${ }^{\mathrm{b}} \mathrm{RI}-\mathrm{ADC}(2) / \mathrm{aug}-\mathrm{cc}-\mathrm{pVTZ}$ results are given in parentheses. 
$5.3 \mathrm{eV}$ for both the RI-ADC(2) and ADC(3) methods when using the def2-SVP basis set, while they are significantly lower in energy in the case of MS-CASPT2 (4.32 and $4.99 \mathrm{eV})$. Interestingly, when the aug-cc-pVTZ basis set is employed, the RI-ADC(2) energies are significantly lower and almost coincide with the MS-CASPT2/def2-SVP energies. The two ${ }^{1} \pi_{S} \pi^{*}$ states are the only states considered here with significant oscillator strengths. All methods agree that the lower ${ }^{1} \pi \pi^{*}$ state has a slightly enhanced oscillator strength compared to the higher energy one. The highest considered state $\left({ }^{1} n_{S} \pi_{6}^{*}\right)$ is an excitation from the $\mathrm{S}$ atom to a $\pi^{*}$ orbital on the opposite side of the ring. At the RI-ADC(2) and ADC(3) levels, this last state possesses enhanced charge-transfer character as indicated by the large dipole moment (above 7.5 D). By contrast, the MS-CASPT2 result shows some admixture with the ${ }^{1} n_{O} \pi_{6}^{*}$ state (cf. Fig. S6 of the supplementary material), which leads to a significantly decreased total dipole moment. It should also be noted that this state possesses substantial contributions of two-electron-excitation character. Considering both MS-CASPT2 and ADC(3), it is found that this state possesses only $72 \%$ of single-excitation character, as measured by the norm of the one-electron transition density matrix. ${ }^{97}$

In the case of the triplet states, the agreement between the three different methods is better. For the first three triplet states, no deviations above $0.25 \mathrm{eV}$ are found among the three methods. The lowest triplet state, located around $3.4 \mathrm{eV}$, is of ${ }^{3} \pi_{S} \pi^{*}$ character and shows similar orbital contributions as the two bright singlet states. Then, two almost degenerate states follow slightly below $4 \mathrm{eV}$ : a ${ }^{3} n_{S} \pi_{2}^{*}$ state of similar character as the $S_{1}$ state and a ${ }^{3} \pi \pi^{*}$ state, delocalized over the whole system, that possesses no direct counterpart among the computed singlet states. In the case of $T_{4}\left({ }^{3} n_{O} \pi_{6}^{*}\right)$ the discrepancies are somewhat bigger, as RI-ADC(2) and MS-CASPT2 place this state at $\approx 4.6 \mathrm{eV}$, while it is at $4.95 \mathrm{eV}$ in the case of $\operatorname{ADC}(3)$. This observation is consistent with the energies of the corresponding singlet state $\left({ }^{1} n_{O} \pi_{6}^{*}\right)$.

Table I shows large discrepancies in the electronic excitations already at the Franck-Condon geometry, even for sophisticated methods as ADC(3) and MS-CASPT2. For the case of $2 \mathrm{TU}$, it is not a priori clear which of these two methods is more reliable, i.e., whether it is more important to move to third order in many-body perturbation theory as in the case of ADC(3) or to include multireference effects as in MS-CASPT2. Moreover, judging the basis set effects is not trivial, considering that MS-CASPT2 with a small basis set and an IPEA shift of zero profits from error compensation, ${ }^{91}$ and hence yields very similar results as MS-CASPT2 with a large CAS $(16,12)$ active space, a quadruple- $\zeta$ basis, and the standard IPEA shift. ${ }^{67}$ Therefore, the results have to be carefully checked individually. On the one hand, it is important to realize that all methods agree satisfactorily in the nature and energy of the $S_{1}$ state as well as the lowest three triplet states. Thus, it can be assumed that the dynamics among these methods will be similar once the $S_{1}$ state is reached and, in particular, that ISC from this state will be described correctly. On the other hand, the energy of the lowest bright state $\left(1^{1} \pi_{S} \pi^{*}\right)$ differs significantly between the ADC methods and MS-CASPT2. In this case, two observations can be made. First, the ADC(2) energy moves closer to the MS-CASPT2 value once a larger basis set is used. Second, the MS-CASPT2 energy is actually too low when compared to the experimental absorption spectrum ${ }^{102}$ (see below). Finally, the possible influence of the ${ }^{1} n_{O} \pi_{6}^{*}$ state on the dynamics has to be examined. This state is placed at a similar energy as the bright state for RI-ADC(2), while it is significantly higher for the other two methods. While this is a potential shortcoming of the RI-ADC(2) method, we notice that this state quickly increases in energy during the dynamics and, thus, does not play a role. We thus summarize that RI-ADC(2)/def2SVP provides a satisfactory description of all relevant states at the Franck-Condon geometry when compared against the reference methods and experiment.

As a next step, we compare the SOC values between the different states as computed with RI-ADC(2) and MSCASPT2. In this case, it should be remembered that a quantitative agreement cannot be expected due to the fact that the wave functions produced by the two methods are not equivalent, as already seen in the $f$ and $\mu$ values shown in Table I. Thus, only a semi-quantitative agreement can be expected. In Table II, the SOC values are collected for the different pairs of states. Both methods agree that the largest SOC value $\left(\approx 125 \mathrm{~cm}^{-1}\right)$ is obtained between the $S_{1}\left({ }^{1} n_{S} \pi_{2}^{*}\right)$ and $T_{1}\left({ }^{3} \pi_{S} \pi^{*}\right)$ states. The second largest value $\left(\approx 100 \mathrm{~cm}^{-1}\right)$ is obtained for the $1^{1} \pi_{S} \pi^{*} /{ }^{3} n_{S} \pi_{2}^{*}$ state pair. Then, the $2^{1} \pi_{S} \pi^{*} /{ }^{3} n_{S} \pi_{2}^{*}$ and ${ }^{1} n_{S} \pi_{2}^{*} / /^{3} \pi \pi^{*}$ follow, both around $75 \mathrm{~cm}^{-1}$. All these values agree reasonably well between the two methods and fall in line with qualitative expectations, i.e., couplings are large between states of different $\left(n \pi^{*}, \pi \pi^{*}\right)$ character and if there are strong contributions on the $\mathrm{S}$ atom.

Recently, Krauter et al..$^{55}$ have evaluated their ADC/SOC code, implemented in Q-ChEM, against the TuRbomole interface used here, finding good agreement for the two molecules investigated (thiophene and 1,2-dithiin). Considering both the results by these authors and the ones in Table II, we conclude that the present SOC implementation for RI-ADC(2) produces accurate results.

\section{B. SHARC dynamics}

In this section, we show that the described RI-ADC(2) method, including the calculation of SOCs and wave function overlaps, can be employed to efficiently carry out accurate SHARc dynamics simulations. To this end, we simulate the excited-state dynamics of $2 \mathrm{TU}$, after excitation to the

TABLE II. Comparison of the SOC terms $\left(\mathrm{cm}^{-1}\right)$ between different singlet and triplet excited states computed at the RI-ADC (2)/def2-SVP and MS $(6,4)$ CASPT2 $(14,10) /$ def2-SVP levels of theory for the MP2-optimized groundstate minimum. ${ }^{\text {a }}$

\begin{tabular}{lcccc}
\hline \hline & ${ }^{3} \pi_{S} \pi^{*}$ & ${ }^{3} n_{S} \pi_{2}^{*}$ & ${ }^{3} \pi \pi^{*}$ & ${ }^{3} n_{O} \pi_{6}^{*}$ \\
\hline${ }^{1} n_{S} \pi_{2}^{*}$ & $\mathbf{1 1 7 / 1 3 1}$ & $0 / 4$ & $\mathbf{6 8 / 7 7}$ & $4 / 1$ \\
${ }^{1} n_{O} \pi_{6}^{*}$ & $3 / 10$ & $4 / 2$ & $32 / 55$ & $1 / 3$ \\
${ }^{1}{ }^{1} \pi_{S} \pi^{*}$ & $0 / 10$ & $\mathbf{1 0 9 / 8 3}$ & $0 / 4$ & $9 / 9$ \\
${ }^{1}{ }^{1} \pi_{S} \pi^{*}$ & $0 / 0$ & $\mathbf{7 3 / 7 6}$ & $0 / 4$ & $30 / 17$ \\
${ }^{1} n_{S} \pi_{6}^{*}$ & $19 / 10$ & $6 / 4$ & $35 / 40$ & $15 / 5$ \\
\hline \hline
\end{tabular}

${ }^{\text {a Results are given in the order RI-ADC(2)/MS-CASPT2. Values above } 60 \mathrm{~cm}^{-1} \text { are }}$ marked in bold. 
lowest bright singlet state $\left(S_{2}\right.$, with $\pi_{S} \pi^{*}$ character in the Franck-Condon region).

The essential parts of the excited-state PESs of 2TU are presented in Fig. 2. In the upper panel of the figure, we present a chain of linear interpolation in internal coordinates (LIIC) scans between the most important critical points, which were individually optimized with each method. These scans can be partitioned into three main relaxation paths. In path I, after excitation to the $S_{2}$ state, the molecule first relaxes to one of its two $S_{2}$ minima, with state character $\pi_{S} \pi_{2}^{*}$ and with a strongly pyramidalized geometry (see the insets). From this minimum, only a small barrier needs to be overcome to reach a $S_{1} / S_{2}$ minimum energy conical intersection (MECI), allowing IC to the $S_{1}\left({ }^{1} n_{S} \pi_{2}^{*}\right.$ character) and relaxation to the $S_{1}$ minimum (pyramidalized geometry). In path II, from the Franck-Condon geometry, the molecule relaxes to the second $S_{2}$ minimum $\left({ }^{1} \pi_{S} \pi_{6}^{*}\right.$ character and nearly planar geometry) and from there can reach another $S_{1} / S_{2}$ MECI, which again allows IC to the $S_{1}$. Path III, which begins in the $S_{1}$ minimum reached by either path I or II, leads to an easily accessible $S_{1} / T_{2}$ minimum energy crossing point (MECP), where sizable SOCs allow for efficient ISC. Once in the $T_{2}$ triplet state, the molecule can relax to one of the two $T_{1}$ minima via a $T_{1} / T_{2}$ MECI. Finally, a second $T_{1}$ minimum can be reached by surpassing an energy barrier.

In the lower panel of Fig. 2, we also show an analogous scan obtained with MS-CASPT2(12,9)/cc-pVDZ, which is the level of theory employed previously for the study of $2 \mathrm{TU} .{ }^{45,67}$ The comparison between RI-ADC(2) and MSCASPT2 reveals that both methods yield qualitatively the same three relaxation pathways as described above. There are some differences between the methods: most importantly, RI$\mathrm{ADC}(2)$ seems to make passage through path I easier and through path II more difficult, compared to MS-CASPT2.
Also, the barrier between the $T_{1}$ minima is larger with RIADC(2) than with MS-CASPT2. However, in general, the agreement is very good. In particular, both methods predict that after excitation the molecule can easily relax from the initial $S_{2}$ state to the $S_{1}$, from where efficient ISC to $T_{2}$, followed by relaxation in the triplet states, can commence.

Encouraged by the good agreement of the MS-CASPT2 and RI-ADC(2) PESs, we went on to perform the SHARC dynamics simulations. The absorption spectrum, which was generated during the initial condition setup, is shown in Fig. 3. The good agreement with the experimental spectrum is another promising indicator of the adequacy of RI-ADC(2) to describe the excited-state dynamics of this molecule.

Figure 4 shows an analysis of the population flow from the RI-ADC(2) dynamics simulations. Since in the dynamics, it is not unambiguously possible to identify the diabatic state character (e.g., $\left.{ }_{1}^{1} n_{S} \pi_{2}^{*}, \ldots\right)$, the following paragraphs discuss the dynamics in terms of the eigenstates of the spin-free Hamiltonian (adiabatic, but spin-diabatic) — note that there is not a one-to-one correspondence to the diabatic states discussed above. Panel (a) shows the time-dependent populations of the excited states. Initially, all population is either in $S_{2}$ or $S_{3}$ (in the figure, their populations are combined as $S_{2,3}$ ), but the population is quickly transferred to the $S_{1}$ state and subsequently to the triplet states. In panel (b), we show the net surface hops between the excited states. As can be seen, most trajectories take a $S_{2,3} \rightarrow S_{1} \rightarrow T_{2,3} \rightarrow T_{1}$ path, as anticipated from the PESs shown in Fig. 2. Hence, $S_{2,3} \rightarrow S_{1} \rightarrow T_{2,3}$ $\rightarrow T_{1}$ would be a good kinetic model to fit the populations shown in Fig. 4(a). However, in order to get time constants consistent with Ref. 45, we use the same kinetic model as in this reference; the model is shown in panel (c). Using this kinetic model, we obtain three time constants describing the population transfer in the trajectories: (i) a time constant of 250

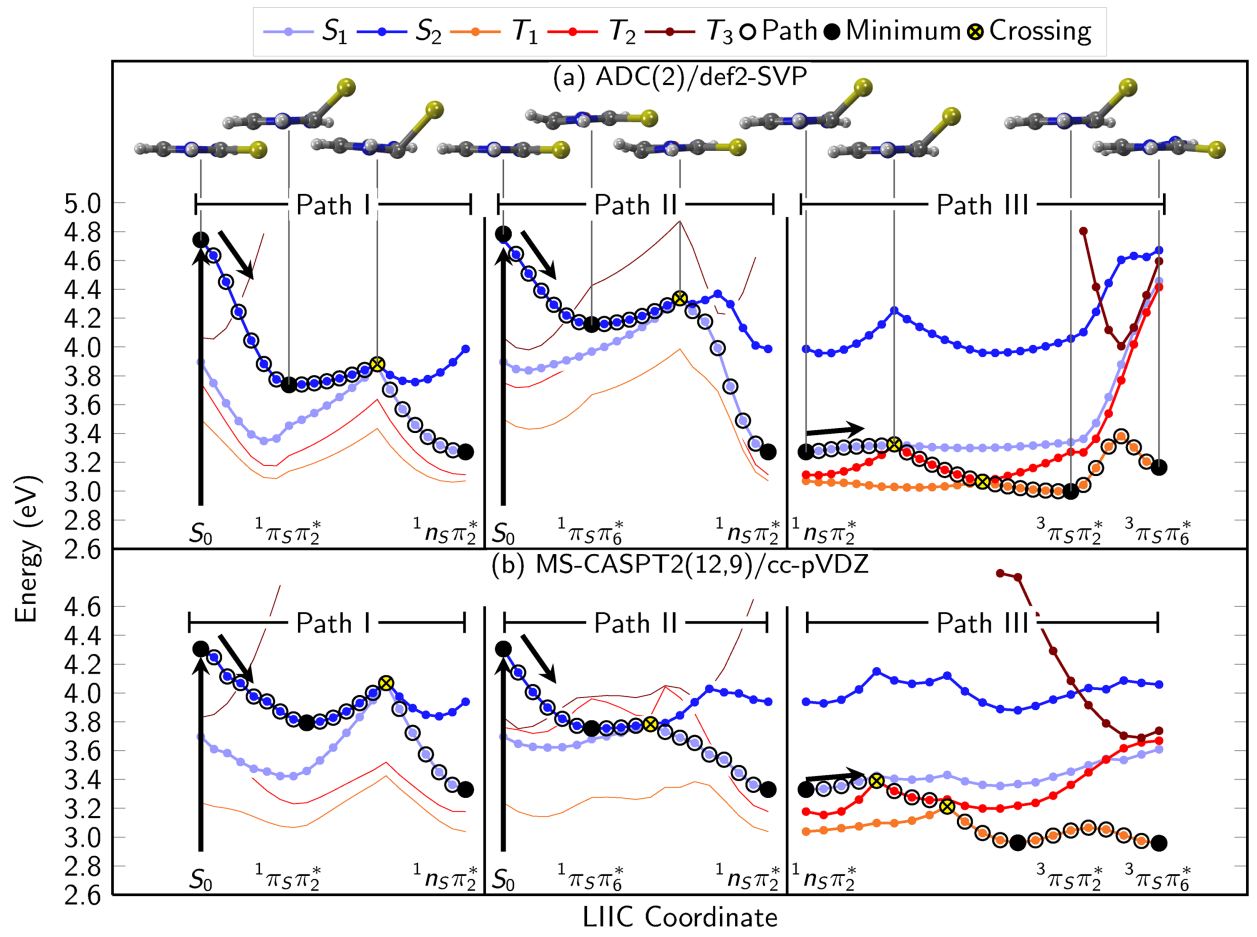

FIG. 2. Linear interpolation in internal coordinates (LIIC) scan for the excited states of $2 \mathrm{TU}$ at the (a) RI-ADC(2)/def2-SVP and (b) MSCASPT2(12,9)/cc-pVDZ levels of theory. (b) was adapted from S. Mai, P. Marquetand, and L. González, J. Phys. Chem. A 119, 9524 (2015). Copyright 2015 Author(s), licensed under a Creative Commons Attribution 4.0 License. 


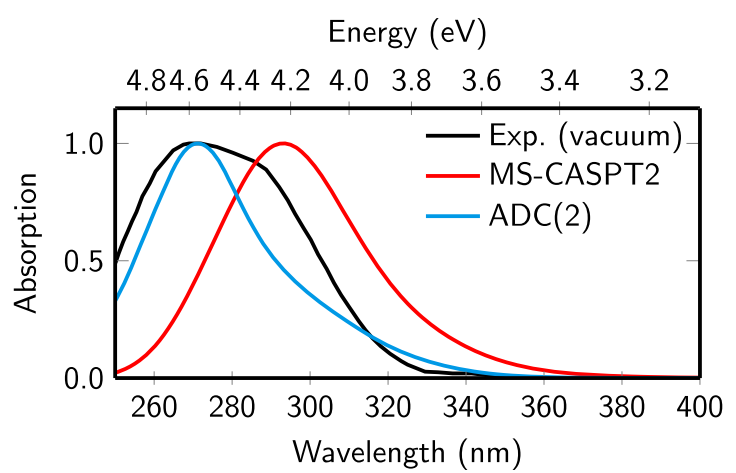

FIG. 3. The experimental vacuum absorption spectrum of $2 \mathrm{TU}^{102}$ together with the absorption spectra simulated with MS-CASPT2 ${ }^{45}$ and RI-ADC(2). All spectra are normalized.

fs for $S_{2,3} \rightarrow S_{1}$ IC, (ii) a constant of 1060 fs for $S_{2,3} \rightarrow T_{2,3}$, and (iii) a time constant of $325 \mathrm{fs}$ for $S_{1} \rightarrow T_{1}$. The corresponding time constants from Ref. 45 are (i) 59 fs, (ii) 250 fs, and (iii) $540 \mathrm{fs}$.

There are some differences to the population flow as reported in Ref. 45. First, with MS-CASPT2, more trajectories hop through the side channels $S_{1} \rightarrow T_{1}$ and $S_{2}$ $\rightarrow T_{2,3}$, while with RI-ADC(2), most trajectories hop according to the main channel $S_{2,3} \rightarrow S_{1} \rightarrow T_{2,3} \rightarrow T_{1}$. Second, with MS-CASPT2, there was a tiny contribution of ground state relaxation from the $S_{1}$, while with RI-ADC(2), ground state relaxation did not take place. Third, with MS-CASPT2, singlet IC is notably faster than with RI-ADC(2) (59 fs versus $250 \mathrm{fs}$ ); this might be due to the different preferences of paths I and II by the two methods. In particular, RI-ADC(2) has similar

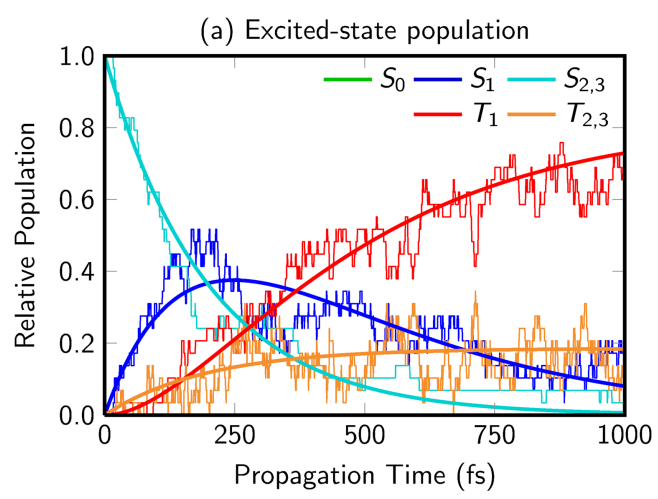

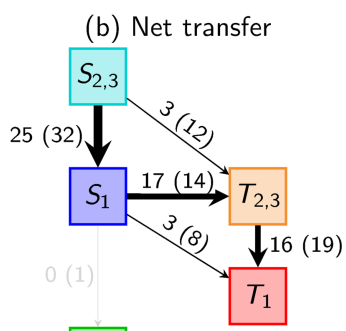

$S_{0}$ (c) Time constants (fs)

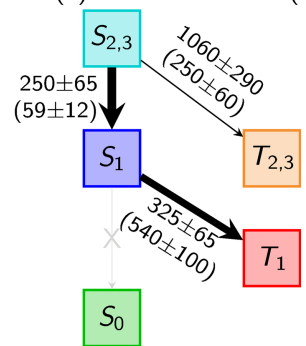

FIG. 4. Excited-state populations, net transfer graph, and fitted time constants for the excited-state dynamics simulations of 2TU with RI-ADC(2)/def2-SVP. Note that the populations of $S_{2}$ and $S_{3}$ as well as $T_{2}$ and $T_{3}$ are combined in the figure. The analysis includes 29 trajectories. In parentheses, the corresponding values from Ref. 45 are given.

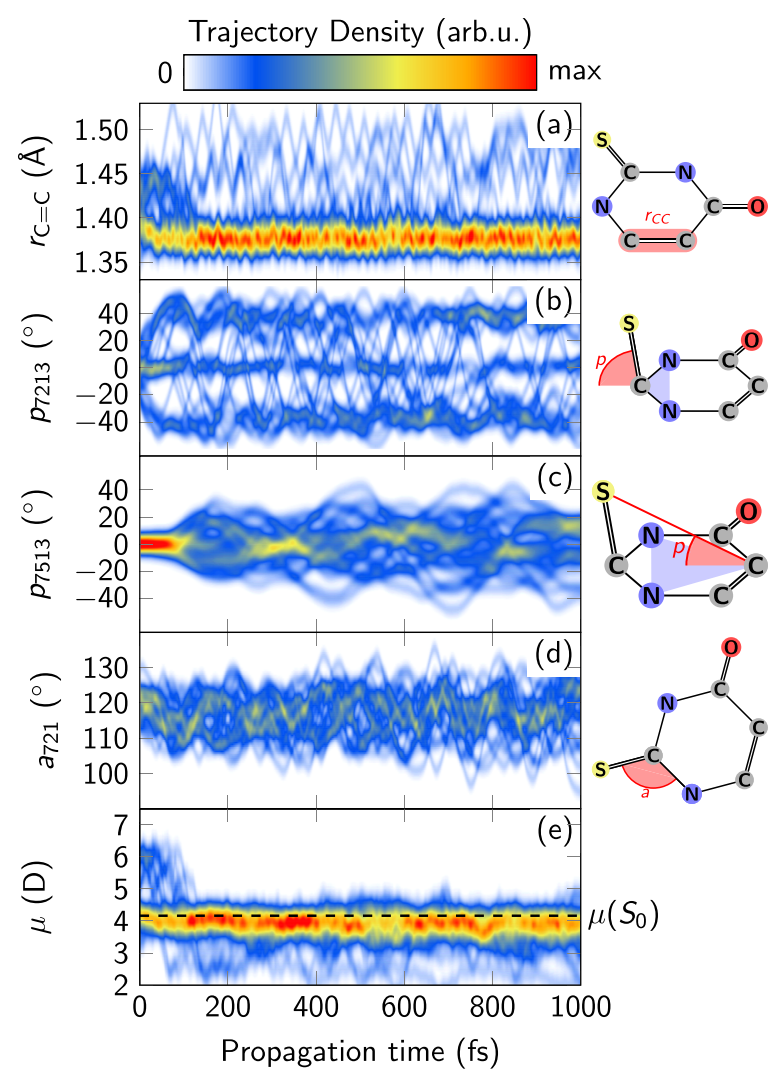

FIG. 5. Time-dependent distribution of internal coordinates of $2 \mathrm{TU}$ with RIADC(2)/def2-SVP. The shown internal coordinate is sketched on the right and highlighted in red.

barriers for both paths, whereas path II at the MS-CASPT2 level is nearly barrierless. Furthermore, with RI-ADC(2), path I involves pyramidalization in $S_{2}$, which takes some time, while with MS-CASPT2, $S_{1}$ can be reached quickly before the slow pyramidalization starts. Fourth, the ISC time constants are different for the two methods. This is partially because the ISC time constants are coupled to the IC time constant (because ISC is a follow-up reaction). As can be seen, the ratio between the $S_{2,3} \rightarrow S_{1}$ and $S_{2,3} \rightarrow T_{2,3}$ time constants is about 1:4 for both electronic structure methods. Hence, after $1000 \mathrm{fs}$, both methods provide very similar populations of the excited states: $65 \% T_{1}, 20 \% T_{2,3}$, and $15 \% S_{1}$ for MS-CASPT2; $70 \%$ $T_{1}, 20 \% T_{2,3}$, and $10 \% S_{1}$ for RI-ADC(2). Thus, it appears that even though the time constants themselves are different, the interplay of the time constants leads to similar overall results.

In Fig. 5, we show an analysis of the molecular motion of 2TU during the simulation, similar to that in Ref. 45. In panel (a), we plot the distribution of the $\mathrm{C}=\mathrm{C}$ bond length over time, which is an indicator mode to distinguish the ${ }^{1} \pi_{S} \pi_{6}^{*}\left(S_{2}\right)$ state from the other states $\left({ }^{1} \pi_{S} \pi_{2}^{*},{ }^{1} n_{S} \pi_{2}^{*}\right.$, and $\left.{ }^{3} \pi_{S} \pi_{2}^{*}\right)$. The former state has a bond length of about $1.44 \AA$, whereas the other states have about $1.37 \AA$. The panel shows clearly that only few trajectories show the long bond typical for the ${ }^{1} \pi_{S} \pi_{6}^{*}$ state, in contrast to the MS-CASPT2 dynamics, where initially all trajectories exhibit the long bond length.

In panel (b), we plot the pyramidalization angle of the thiocarbonyl group, one of the most important modes in the 
excited-state dynamics of 2TU. The plot shows that pyramidalization starts immediately after excitation; in MS-CASPT2, pyramidalization only starts after about $50 \mathrm{fs}$, when the trajectories change from the planar ${ }^{1} \pi_{S} \pi_{6}^{*}$ state to the pyramidalized ${ }^{1} n_{S} \pi_{2}^{*}$ state. The plots in panels (a) and (b) unambiguously show that RI-ADC(2) prefers path I (see Fig. 2) over path II, in opposition to MS-CASPT2.

Panel (c) shows another pyramidalization angle, which measures how much the sulfur atom is displaced from the ring plane while ignoring the motion of the thiocarbonyl carbon atom. This panel is in good agreement with the MS-CASPT2 dynamics, showing that the long-term motion of the molecule in the $S_{1}$ and $T_{1}$ states is correctly captured. The same can be said for panel (d), which shows the bond angle which controls ISC by tuning the energy gap between the ${ }^{1} n_{S} \pi_{2}^{*}$ and ${ }^{3} \pi_{S} \pi_{2}^{*}$ states, which are primarily involved in the ISC process.

Finally, in panel (e), we plot the temporal evolution of the permanent dipole moment of 2TU. Like panels (a) and (b), this panel also shows that RI-ADC(2) favors path I, as the more polar ${ }^{1} \pi_{S} \pi_{6}^{*}$ state (at about $6 \mathrm{D}$ ) is not strongly populated at early times. Moreover, the panel shows that RI-ADC(2) provides very similar permanent dipole moments for the other excited states, which are all around 4.0 D and similar to the ground state dipole moment of $4.2 \mathrm{D}$.

As a brief remark, three $\mathrm{ADC}(2)$ trajectories followed a ring-opening pathway, with the bond between N3 and C4 breaking. Additional calculations showed that the relevant barrier is about 4.0-4.2 eV above the ground state energy [with both ADC(2) and MS-CASPT2] and hence much higher than the $S_{1}$ and $T_{1}$ minima. Consequently, the three trajectories all undergo ring opening from the $S_{2}$ state briefly after excitation. The reason for the more frequent ring opening with $\mathrm{ADC}(2)$ compared to MS-CASPT2 ${ }^{45}$ might be that ADC(2) predicts rather high vertical excitation energies and thus an increased initial energy, enough to overcome the ring opening barrier. Still, as mentioned above, we neglected the ring-opening trajectories from the above analysis, as the $S_{0}-S_{1}$ energy gap becomes very small and $\mathrm{ADC}(2)$ is not expected to be reliable in this situation.

\section{Computational remarks}

After the discussion of the dynamics simulations results, here we would also like to mention the computational effort involved in the present calculations. Each of the RI$\mathrm{ADC}(2) / \mathrm{def} 2-\mathrm{SVP}$ trajectories calculated was run on 2 cores of an Intel ${ }^{\circledR}$ Xeon E5-2650-v3 CPU and the 2000 time steps were on average finished after 5 days, which is equivalent to 240 core hours per trajectory. By comparison, the MS-CASPT2 trajectories reported in Ref. 45 were run on 16 cores of an Intel ${ }^{\circledR}$ Xeon E5-2650-v2 CPU, and completion of 2000 time steps took about 18000 core hours per trajectory. Considering also that the MS-CASPT2 trajectories included one state less (the $S_{3}$ ) and that due to convergence problems, a sizable fraction of the MS-CASPT2 trajectories had to be neglected, it appears that RI-ADC(2) was over 100 times more CPU-time-efficient than MS-CASPT2 for the chosen molecule. Due to the scaling behaviors of the two methods, it can be expected that for larger systems, RI-ADC(2) will be even more favorable. Furthermore, RI-ADC(2) offers advantages due to its conceptual simplicity owing to the fact that no active space has to be selected; this not only makes the computations more user friendly but also offers more stable convergence behavior along a trajectory and allows studying systems where many relevant orbitals are involved.

\section{CONCLUSIONS}

In this contribution, a new implementation is reported which allows performing surface hopping simulations of ISC processes in the SHARC dynamics package in combination with the $a b$ initio RI-ADC(2) electronic structure methoda computationally efficient, reliable, and easy-to-use method. We expect that this new implementation will provide a powerful tool for simulating ISC between one-electron excited states in a large variety of organic molecules. For completeness, it should be pointed out that RI-ADC(2) is not expected to work in some special cases involving two-electron excited states, ${ }^{103}$ transition metal complexes, ${ }^{104}$ or strongly distorted geometries. Furthermore, RI-ADC(2) cannot correctly describe conical intersections between the ground state $\left(S_{0}\right)$ and the excited states. In such cases, the user is advised to use multi-reference methods, e.g., as those already available in SHARC. ${ }^{45,47}$

The 2-thiouracil molecule was chosen to demonstrate the capabilities of the new implementation. First, the vertical excitations were compared to the more sophisticated ADC(3) and MS-CASPT2 methods. While this comparison showed some differences between the methods, RI-ADC(2) provided an excellent description of all the states relevant for the dynamics of this molecule. Furthermore, the SOC terms were compared between the RI-ADC(2) and MS-CASPT2 methods, showing satisfactory agreement. Finally, RI-ADC(2) dynamics simulations were performed and compared to previously reported MS-CASPT2 results. ${ }^{45}$ Slightly different mechanistic details were obtained as $S_{2} \rightarrow S_{1}$ IC is accompanied at the RI-ADC(2) level by pyramidalization in the $S_{2}$ state, whereas MS-CASPT2 predicts that pyramidalization is initiated in the $S_{1}$ state. Nevertheless, good agreement between both methods is found in terms of the main deactivation mechanisms, i.e., IC to the $S_{1}$ state is followed by ISC, which leads to the population of the $T_{1}$ state in less than 1 ps.

\section{SUPPLEMENTARY MATERIAL}

See supplementary material for coordinates of the geometry and active space orbitals used for the results presented in Sec. IV A and natural transition orbitals of $2 \mathrm{TU}$ for the RI-ADC(2), ADC(3), and MS-CASPT2 calculations in Sec. IV A.

\section{ACKNOWLEDGMENTS}

S.M., F.P., and L.G. thank the Austrian Science Fund (FWF) for funding through Project No. P25827, the University 
of Vienna, and the Vienna Scientific Cluster (VSC3) for generous allocation of computing time. A.K. and M.P. acknowledge generous support by Professor J. Gauss during their stay in Mainz. A.K. also thanks Professor M. Kallay for helpful discussions about ADC(2) transition densities. The authors also acknowledge the COST action CM1305 (ECOSTBio).

\section{APPENDIX: SPIN-FREE FORMULATION OF TRIPLET TRANSITION DENSITIES}

The implementation in TuRbomole allows for the computation of all spin-orbit matrix elements in both the spin-orbital basis and a spin-free formulation. As the spin-orbital formulation does not offer explicit control of the spin state, its use for open-shell ground states is not recommended. For closedshell ground states, a more efficient spin-free formulation for both singlet and triplet excited states is available, which has been tested against the spin-orbital code. The spin-free cluster operator is defined as

$$
\hat{T}_{2}=\frac{1}{2} \sum_{i j a b} t_{i a j b} \hat{E}_{a i} \hat{E}_{b j},
$$

where

$$
t_{i a j b}=-\frac{g_{i a j b}}{\epsilon_{a}+\epsilon_{b}-\epsilon_{i}-\epsilon_{j}}
$$

are the first-order amplitudes from MP2 theory. These are computed from two-electron repulsion integrals $g_{i a j b}$ and canonical orbital energies $\epsilon_{p}$.

The singlet excited state $I$ is associated with the excitation operator $\hat{R}^{I}=\hat{R}_{1}^{I}+\hat{R}_{2}^{I}$, with single and double excitations

$$
\begin{aligned}
& \hat{R}_{1}^{I}=\sum_{i a} R_{i a}^{I} \hat{E}_{a i}, \\
& \hat{R}_{2}^{I}=\frac{1}{2} \sum_{i j a b} R_{i a j b}^{I} \hat{E}_{a i} \hat{E}_{b j} .
\end{aligned}
$$

For the formulation of triplet excited states, we introduce the triplet excitation operator $\hat{T}_{a i}=2 \hat{E}_{a i}^{z}=\hat{a}_{a}^{\dagger} \hat{a}_{i}-\hat{a}_{\bar{a}}^{\dagger} \hat{a}_{\bar{i}}$ and define the single and double excitations as

$$
\begin{aligned}
{ }^{3} \hat{R}_{1}^{I}= & \sum_{i a}{ }^{3} R_{i a}^{I} \hat{T}_{a i}, \\
{ }^{3} \hat{R}_{2}^{I}= & \sum_{i j a b}{ }^{3} R_{i a j b}^{I} \hat{T}_{a i} \hat{E}_{b j} \\
= & \frac{1}{4} \sum_{i j a b}{ }^{(+)} R_{i a j b}^{I}\left(\hat{T}_{a i} \hat{E}_{b j}+\hat{E}_{a i} \hat{T}_{b j}\right) \\
& +\frac{1}{2} \sum_{i j a b}{ }^{(-)} R_{i a j b}^{I}\left(\hat{T}_{a i} \hat{E}_{b j}-\hat{E}_{a i} \hat{T}_{b j}\right) .
\end{aligned}
$$

The symmetrized formulation has been given for comparison with the triplet basis introduced in Refs. 105 and 106. The ADC(2) equations using the singlet and triplet adapted excitation operators can be easily derived from the more general CC2 equations given in Refs. 60, 74, 105, and 106.

We only want to explicitly quote the spin-transition densities from ground to excited states, $D_{p q}^{0 J, z}=\left\langle\Psi_{0}\left|\hat{E}_{p q}^{z}\right| \Psi_{J}\right\rangle$, and between excited states, $D_{p q}^{I J, z}=\left\langle\Psi_{I}\left|\hat{E}_{p q}^{z}\right| \Psi_{J}\right\rangle$. The ground-toexcited-state transition density reads

$$
\begin{aligned}
& D_{i j}^{0 J, z}=-\frac{1}{\sqrt{2}} \sum_{c d l} t_{c j d l}^{\dagger}{ }^{3} R_{i c l d}^{J}, \\
& D_{i a}^{0 J, z}=\frac{1}{\sqrt{2}}{ }^{3} R_{i a}^{J}, \\
& D_{a i}^{0 J, z}=-\frac{1}{\sqrt{2}} \sum_{c k} t_{c i a k}^{\dagger}{ }^{3} R_{k c}^{J}, \\
& D_{a b}^{0 J, z}=\frac{1}{\sqrt{2}} \sum_{d k l} t_{a k d l}^{\dagger}{ }^{3} R_{k b l d}^{J},
\end{aligned}
$$

and the transition density between excited states is given by

$$
\begin{aligned}
D_{i j}^{I J, z}= & \frac{1}{2}\left[-\sum_{c} R_{c j}^{I \dagger 3} R_{i c}^{J}-\sum_{c d l} R_{c j d l}^{I \dagger}{ }^{3} R_{i c l d}^{J}\right] \\
D_{i a}^{I J, z}= & \frac{1}{2}\left[\sum_{d l} R_{d l}^{I \dagger 3} R_{i a l d}^{J}-\sum_{c d k l}\left[2 R_{c k d l}^{I \dagger}-R_{d k c l}^{I \dagger}\right] t_{k a l d}{ }^{3} R_{i c}^{J}\right. \\
& \left.-\sum_{c d k l}\left[2 R_{c k d l}^{I \dagger}-R_{d k c l}^{I \dagger}\right] t_{i c l d}^{3} R_{k a}^{J}\right] \\
D_{a i}^{I J, z}= & \frac{1}{2}\left[-\sum_{d l} R_{d i a l}^{I \dagger} R_{l d}^{J}-\sum_{c d k l} R_{c i}^{I \dagger} t_{a k d l}^{\dagger}{ }^{3} R_{k c l d}^{J}\right. \\
& \left.-\sum_{c d k l} R_{a k}^{I \dagger} t_{c k d l}^{\dagger}{ }^{3} R_{i c l d}^{J}\right] \\
D_{a b}^{I J, z}= & \frac{1}{2}\left[\sum_{k} R_{a k}^{I \dagger 3} R_{k b}^{J}+\sum_{d k l} R_{a k d l}^{I \dagger}{ }^{3} R_{k b l d}^{J}\right]
\end{aligned}
$$

In the actual implementation, all four-index quantities are on-the-fly recomputed from three-index quantities using the resolution-of-the-identity trick as described in Refs. 74 and 106.

${ }^{1}$ M. Klessinger and J. Michl, Excited States and Photochemistry of Organic Molecules (VCH Publishers Inc., New York, 1995).

${ }^{2}$ N. J. Turro, J. C. Scaiano, and V. Ramamurthy, Principles of Molecular Photochemistry: An Introduction (University Science Books, Sausalito, CA, 2009).

${ }^{3}$ J. L. Segura, N. Martin, and D. M. Guldi, Chem. Soc. Rev. 34, 31 (2005).

${ }^{4}$ M. T. Colvin, A. B. Ricks, A. M. Scott, A. L. Smeigh, R. Carmieli, T. Miura, and M. R. Wasielewski, J. Am. Chem. Soc. 133, 1240 (2011).

${ }^{5}$ H. Uoyama, K. Goushi, K. Shizu, H. Nomura, and C. Adachi, Nature 492, 234 (2012).

${ }^{6}$ A. J. Epstein, MRS Bull. 28, 492 (2003).

${ }^{7}$ P. Gütlich, A. Hauser, and H. Spiering, Angew. Chem., Int. Ed. Engl. 33, 2024 (1994).

${ }^{8}$ S. Roy, C. P. Singh, and K. P. J. Reddy, Appl. Phys. Lett. 77, 2656 (2000). ${ }^{9}$ T. Saito and W. Thiel, J. Phys. Chem. B 118, 5034 (2014).

${ }^{10} \mathrm{M}$. Barbatti, A. C. Borin, and S. Ullrich, in Photoinduced Phenomena in Nucleic Acids I, Topics in Current Chemistry Vol. 355, edited by M. Barbatti, A. C. Borin, and S. Ullrich (Springer Berlin Heidelberg, 2015), pp. 1-32.

${ }^{11}$ D. E. J. G. J. Dolmans, D. Fukumura, and R. K. Jain, Nat. Rev. Cancer 3, 380 (2003).

${ }^{12} \mathrm{H}$. Yersin, Transition Metal and Rare Earth Compounds III: Excited States, Transitions, Interactions (Springer Berlin Heidelberg, 2004), Vol. 241, pp. $1-26$.

${ }^{13}$ J. K. McCusker, Acc. Chem. Res. 36, 876 (2003).

${ }^{14}$ L. S. Forster, Coord. Chem. Rev. 250, 2023 (2006).

${ }^{15}$ A. Vlček, "Ultrafast excited-state processes in Re(I) carbonyl-diimine complexes: From excitation to photochemistry," in Photophysics of Organometallics, edited by A. J. Lees (Springer Berlin Heidelberg, Berlin, Heidelberg, 2010), pp. 115-158. 
${ }^{16}$ A. Cannizzo, C. Milne, C. Consani, W. Gawelda, C. Bressler, F. van Mourik, and M. Chergui, Coord. Chem. Rev. 254, 2677 (2010).

${ }^{17}$ G. Auböck and M. Chergui, Nat. Chem. 7, 629 (2015).

${ }^{18}$ S. Aloïse, C. Ruckebusch, L. Blanchet, J. Réhault, G. Buntinx, and J.-P. Huvenne, J. Phys. Chem. A 112, 224 (2008).

${ }^{19}$ M. Huix-Rotllant, D. Siri, and N. Ferré, Phys. Chem. Chem. Phys. 15, 19293 (2013)

${ }^{20}$ T. Kobayashi, H. Kuramochi, Y. Harada, T. Suzuki, and T. Ichimura, J. Phys. Chem. A 113, 12088 (2009).

${ }^{21}$ S. Matsika, in Photoinduced Phenomena in Nucleic Acids I, Topics in Current Chemistry Vol. 355, edited by M. Barbatti, A. C. Borin, and S. Ullrich (Springer Berlin Heidelberg, 2015), pp. 209-243.

${ }^{22}$ M. Pollum, L. Martínez-Fernández, and C. E. Crespo-Hernández, in Photoinduced Phenomena in Nucleic Acids I, Topics in Current Chemistry Vol. 355, edited by M. Barbatti, A. C. Borin, and S. Ullrich (Springer Berlin Heidelberg, 2015), pp. 245-327.

${ }^{23}$ K. G. Dyall and K. Fægri, Introduction to Relativistic Quantum Chemistry (Oxford University Press, 2007).

${ }^{24}$ M. Reiher and A. Wolf, Relativistic Quantum Chemistry (Wiley VCH Verlag, Weinheim, 2009)

${ }^{25}$ C. M. Marian, Wiley Interdiscip. Rev.: Comput. Mol. Sci. 2, 187 (2012).

${ }^{26}$ J. Eng, C. Gourlaouen, E. Gindensperger, and C. Daniel, Acc. Chem. Res. 48, 809 (2015).

${ }^{27}$ M. Fumanal, E. Gindensperger, and C. Daniel, J. Chem. Theory Comput. 13, 1293 (2017)

${ }^{28}$ G. Capano, T. J. Penfold, M. Chergui, and I. Tavernelli, Phys. Chem. Chem. Phys. 19, 19590 (2017).

${ }^{29}$ J. C. Tully, J. Chem. Phys. 93, 1061 (1990)

${ }^{30}$ M. Barbatti, Wiley Interdiscip. Rev.: Comput. Mol. Sci. 1, 620 (2011).

${ }^{31}$ G. Granucci, M. Persico, and G. Spighi, J. Chem. Phys. 137, 22A501 (2012).

${ }^{32}$ G. Cui and W. Thiel, J. Chem. Phys. 141, 124101 (2014).

${ }^{33}$ S. Mai, P. Marquetand, and L. González, Int. J. Quantum Chem. 115, 1215 (2015).

${ }^{34}$ F. Franco de Carvalho and I. Tavernelli, J. Chem. Phys. 143, 224105 (2015).

${ }^{35}$ B. F. E. Curchod, C. Rauer, P. Marquetand, L. González, and T. J. Martínez, J. Chem. Phys. 144, 101102 (2016)

${ }^{36}$ M. Richter, P. Marquetand, J. González-Vázquez, I. Sola, and L. González, J. Chem. Theory Comput. 7, 1253 (2011).

${ }^{37}$ S. Mai, M. Richter, M. Ruckenbauer, M. Oppel, P. Marquetand, and L. González, "SHARC: Surface hopping including arbitrary couplingsprogram package for non-adiabatic dynamics," sharc-md.org (2014).

${ }^{38}$ L. Martínez-Fernández, I. Corral, G. Granucci, and M. Persico, Chem. Sci. 5, 1336 (2014).

${ }^{39}$ L. Favero, G. Granucci, and M. Persico, Phys. Chem. Chem. Phys. 18, 10499 (2016).

${ }^{40}$ M. Richter, S. Mai, P. Marquetand, and L. González, Phys. Chem. Chem. Phys. 16, 24423 (2014).

${ }^{41}$ M. Marazzi, S. Mai, D. Roca-Sanjuán, M. G. Delcey, R. Lindh, L. González, and A. Monari, J. Phys. Chem. Lett. 7, 622 (2016).

${ }^{42}$ M. Pederzoli and J. Pittner, J. Chem. Phys. 146, 114101 (2017).

${ }^{43}$ D. Bellshaw, D. A. Horke, A. D. Smith, H. M. Watts, E. Jager, E. Springate, O. Alexander, C. Cacho, R. T. Chapman, A. Kirrander, and R. S. Minns, Chem. Phys. Lett. 683, 383 (2017).

${ }^{44}$ H. Tao, B. G. Levine, and T. J. Martínez, J. Phys. Chem. A 113, 13656 (2009)

${ }^{45}$ S. Mai, P. Marquetand, and L. González, J. Phys. Chem. Lett. 7, 1978 (2016)

${ }^{46}$ J. W. Park and T. Shiozaki, J. Chem. Theory Comput. 13, 3676 (2017).

${ }^{47}$ S. Mai, P. Marquetand, and L. González, J. Chem. Phys. 140, 204302 (2014).

${ }^{48}$ S. Mai, M. Pollum, L. Martínez-Fernández, N. Dunn, P. Marquetand, I. Corral, C. E. Crespo-Hernández, and L. González, Nat. Commun. 7, 13077 (2016)

${ }^{49}$ A. C. Borin, S. Mai, P. Marquetand, and L. González, Phys. Chem. Chem. Phys. 19, 5888 (2017).

${ }^{50}$ F. Peccati, S. Mai, and L. González, Philos. Trans. R. Soc., A 375, 20160202 (2017)

${ }^{51}$ A. J. Atkins and L. González, J. Phys. Chem. Lett. 8, 3840 (2017)

52 "TURBOMOLE v7.0, A development of University of Karlsruhe and forschungszentrum karlsruhe GmbH," 2015.

${ }^{53}$ F. Plasser, R. Crespo-Otero, M. Pederzoli, J. Pittner, H. Lischka, and M. Barbatti, J. Chem. Theory Comput. 10, 1395 (2014).
${ }^{54}$ M. Pabst, "Übergangsmomente zwischen angeregten zuständen mit der RICC2 methode: Implementierung und anwendung auf triplett-excimere," $\mathrm{Ph}$.D. thesis, Johannes Gutenberg-Universität in Mainz, 2011.

${ }^{55}$ C. M. Krauter, B. Schimmelpfennig, M. Pernpointner, and A. Dreuw, Chem. Phys. 482, 286 (2017), electrons and nuclei in motion - correlation and dynamics in molecules (on the occasion of the 70th birthday of Lorenz S. Cederbaum).

${ }^{56}$ B. Helmich-Paris, C. Hättig, and C. van Wüllen, J. Chem. Theory Comput. 12, 1892 (2016).

${ }^{57}$ S. Knippenberg, D. R. Rehn, M. Wormit, J. H. Starcke, I. L. Rusakova, A. B. Trofimov, and A. Dreuw, J. Chem. Phys. 136, 064107 (2012).

${ }^{58}$ J. Schirmer and A. B. Trofimov, J. Chem. Phys. 120, 11449 (2004).

${ }^{59}$ M. Pabst and A. Köhn, J. Chem. Phys. 129, 214101 (2008).

${ }^{60} \mathrm{C}$. Hättig and F. Weigend, J. Chem. Phys. 113, 5154 (2000).

${ }^{61}$ X. Gao, S. Bai, D. Fazzi, T. Niehaus, M. Barbatti, and W. Thiel, J. Chem. Theory Comput. 13, 515 (2017).

${ }^{62}$ M. Pollum and C. E. Crespo-Hernández, J. Chem. Phys. 140, 071101 (2014).

${ }^{63}$ H. Yu, J. A. Sánchez-Rodríguez, M. Pollum, C. E. Crespo-Hernández, S. Mai, P. Marquetand, L. González, and S. Ullrich, Phys. Chem. Chem. Phys. 18, 20168 (2016)

${ }^{64}$ J. A. Sanchez-Rodriguez, A. Mohamadzade, S. Mai, B. Ashwood, M. Pollum, P. Marquetand, L. González, C. E. Crespo-Hernández, and S. Ullrich, Phys. Chem. Chem. Phys. 19, 19756 (2017).

${ }^{65}$ G. Cui and W.-H. Fang, J. Chem. Phys. 138, 044315 (2013).

${ }^{66}$ J. P. Gobbo and A. C. Borin, Comput. Theor. Chem. 1040-1041, 195 (2014)

${ }^{67}$ S. Mai, P. Marquetand, and L. González, J. Phys. Chem. A 119, 9524 (2015).

${ }^{68}$ L. Verlet, Phys. Rev. 159, 98 (1967).

${ }^{69}$ G. Granucci, M. Persico, and A. Toniolo, J. Chem. Phys. 114, 10608 (2001)

${ }^{70}$ F. Plasser, G. Granucci, J. Pittner, M. Barbatti, M. Persico, and H. Lischka, J. Chem. Phys. 137, 22A514 (2012).

${ }^{71}$ J. Schirmer, Phys. Rev. A 26, 2395 (1982)

${ }^{72}$ A. B. Trofimov and J. Schirmer, J. Phys. B: At., Mol. Opt. Phys. 28, 2299 (1995).

${ }^{73}$ C. Hättig, Adv. Quantum Chem. 50, 37 (2005).

${ }^{74}$ C. Hättig and A. Köhn, J. Chem. Phys. 117, 6939 (2002).

${ }^{75}$ A. Berning, M. Schweizer, H. Werner, P. J. Knowles, and P. Palmieri, Mol Phys. 98, 1823 (2000).

${ }^{76}$ F. Neese, J. Chem. Phys. 122, 034107 (2005).

${ }^{77}$ S. Hammes-Schiffer and J. C. Tully, J. Chem. Phys. 101, 4657 (1994).

${ }^{78}$ E. Tapavicza, I. Tavernelli, and U. Rothlisberger, Phys. Rev. Lett. 98 023001 (2007)

${ }^{79}$ R. Mitrić, U. Werner, and V. Bonačić-Koutecký, J. Chem. Phys. 129, 164118 (2008)

${ }^{80}$ J. Pittner, H. Lischka, and M. Barbatti, Chem. Phys. 356, 147 (2009).

${ }^{81}$ F. Plasser, M. Ruckenbauer, S. Mai, M. Oppel, P. Marquetand, and L. González, J. Chem. Theory Comput. 12, 1207 (2016).

${ }^{82}$ P. H. P. Harbach, M. Wormit, and A. Dreuw, J. Chem. Phys. 141, 064113 (2014).

${ }^{83}$ A. Dreuw and M. Wormit, Wiley Interdiscip. Rev.: Comput. Mol. Sci. 5, $82(2015)$

${ }^{84}$ K. Andersson, P.-Å. Malmqvist, B. O. Roos, A. J. Sadlej, and K. Wolinski, J. Phys. Chem. 94, 5483 (1990).

${ }^{85}$ F. Weigend and R. Ahlrichs, Phys. Chem. Chem. Phys. 7, 3297 (2005).

${ }^{86}$ T. H. Dunning, J. Chem. Phys. 90, 1007 (1989).

${ }^{87}$ F. Neese, Wiley Interdiscip. Rev.: Comput. Mol. Sci. 2, 73 (2012).

${ }^{88}$ Y. Shao, L. F. Molnar, Y. Jung, J. Kussmann, C. Ochsenfeld, S. T. Brown, A. T. Gilbert, L. V. Slipchenko, S. V. Levchenko, D. P. O’Neill, R. A. DiStasio, Jr., R. C. Lochan, T. Wang, G. J. Beran, N. A. Besley, J. M. Herbert, C. Yeh Lin, T. Van Voorhis, S. Hung Chien, A. Sodt, R. P. Steele, V. A. Rassolov, P. E. Maslen, P. P. Korambath, R. D. Adamson, B. Austin, J. Baker, E. F. C. Byrd, H. Dachsel, R. J. Doerksen, A. Dreuw, B. D. Dunietz, A. D. Dutoi, T. R. Furlani, S. R. Gwaltney, A. Heyden, S. Hirata, C.-P. Hsu, G. Kedziora, R. Z. Khalliulin, P. Klunzinger, A. M. Lee, M. S. Lee, W. Liang, I. Lotan, N. Nair, B. Peters, E. I. Proynov, P. A. Pieniazek, Y. Min Rhee, J. Ritchie, E. Rosta, C. David Sherrill, A. C. Simmonett, J. E. Subotnik, H. Lee Woodcock III, W. Zhang, A. T. Bell, A. K. Chakraborty, D. M. Chipman, F. J. Keil, A. Warshel, W. J. Hehre, H. F. Schaefer III, J. Kong, A. I. Krylov, P. M. W. Gill, and M. Head-Gordon, Phys. Chem. Chem. Phys. 8, 3172 (2006).

${ }^{89}$ F. Aquilante, J. Autschbach, R. K. Carlson, L. F. Chibotaru, M. G. Delcey, L. De Vico, I. Fdez. Galván, N. Ferré, L. M. Frutos, L. Gagliardi, M. Garavelli, A. Giussani, C. E. Hoyer, G. Li Manni, H. Lischka, D. Ma, 
P.-Å. Malmqvist, T. Müller, A. Nenov, M. Olivucci, T. B. Pedersen, D. Peng, F. Plasser, B. Pritchard, M. Reiher, I. Rivalta, I. Schapiro, J. Segarra-Martí, M. Stenrup, D. G. Truhlar, L. Ungur, A. Valentini, S. Vancoillie, V. Veryazov, V. P. Vysotskiy, O. Weingart, F. Zapata, and R. Lindh, J. Comput. Chem. 37, 506 (2015).

${ }^{90}$ B. O. Roos, P. R. Taylor, and P. E. M. Siegbahn, Chem. Phys. 48, 157 (1980).

${ }^{91}$ J. P. Zobel, J. J. Nogueira, and L. González, Chem. Sci. 8, 1482 (2017).

${ }^{92}$ G. Ghigo, B. O. Roos, and P.-Å. Malmqvist, Chem. Phys. Lett. 396, 142 (2004).

${ }^{93}$ P.-Å. Malmqvist, B. O. Roos, and B. Schimmelpfennig, Chem. Phys. Lett. 357, 230 (2002).

${ }^{94}$ B. A. Heß, C. M. Marian, U. Wahlgren, and O. Gropen, Chem. Phys. Lett. 251, 365 (1996).

${ }^{95}$ R. L. Martin, J. Chem. Phys. 118, 4775 (2003).

${ }^{96}$ F. Plasser and H. Lischka, J. Chem. Theory Comput. 8, 2777 (2012).

${ }^{97}$ F. Plasser, M. Wormit, and A. Dreuw, J. Chem. Phys. 141, 024106 (2014).
${ }^{98}$ F. Plasser, "TheoDORE 1.4: A package for theoretical density, orbital relaxation, and exciton analysis," http://theodore-qc.sourceforge.net (2017).

${ }^{99}$ F. Plasser, S. A. Mewes, A. Dreuw, and L. González, "Detailed wavefunction analysis for multireference methods: Implementation in the MoLcAS program package and applications to tetracene" J. Chem. Theory Comput. (published online).

${ }^{100}$ M. Barbatti, G. Granucci, M. Persico, M. Ruckenbauer, M. Vazdar, M. Eckert-Maksic, and H. Lischka, J. Photochem. Photobiol. A 190, 228 (2007).

${ }^{101}$ G. Granucci and M. Persico, J. Chem. Phys. 126, 134114 (2007).

${ }^{102}$ A. Khvorostov, L. Lapinski, H. Rostkowska, and M. J. Nowak, J. Phys. Chem. A 109, 7700 (2005)

${ }^{103}$ J. H. Starcke, M. Wormit, J. Schirmer, and A. Dreuw, Chem. Phys. 329, 39 (2006).

${ }^{104}$ F. Plasser and A. Dreuw, J. Phys. Chem. A 119, 1023 (2015).

${ }^{105}$ K. Hald, C. Hättig, D. L. Yeager, and P. Jørgensen, Chem. Phys. Lett. 328, 291 (2000).

${ }^{106}$ C. Hättig, A. Köhn, and K. Hald, J. Chem. Phys. 116, 5401 (2002). 